\title{
A ABORDAGEM DAS EMOÇÕES NO PROJETO DE MATERIAIS EDUCACIONAIS MULTIMÍDIA
}

Kelly Cristina Bidone Pinto

Universidade Federal do Rio Grande do Sul

kelly.bidone@gmail.com

Gabriela Zubaran de Azevedo Pizzato

Universidade Federal do Rio Grande do Sul

gabriela.zubaran@ufrgs.br

Régio Pierre da Silva

Universidade Federal do Rio Grande do Sul

regio@ufrgs.br

Resumo: As emoções estão presentes em todas as atividades do cotidiano, incluindo a aprendizagem. Este artigo apresenta uma revisão sistemática da literatura sobre a relação entre as emoções e a aprendizagem para o projeto de materiais educacionais multimídia. A partir das bases de dados Web of Science e Periódicos CAPES, foram pesquisados artigos publicados internacionalmente, no período de 2010 a 2015. Após a análise dos resultados, verificou-se que foram poucos os trabalhos desenvolvidos neste período, porém de relevância, visto que, de modo geral, os estudos confirmaram a influência das emoções na aprendizagem e a importância de suscitar emoções que motivem os alunos favorecendo o uso de sua capacidade cognitiva da melhor forma possível. Nestes artigos, é indicada a importância e a influência dos elementos gráfico-visuais dos materiais, pois, uma vez que esses elementos despertam emoções nos alunos, concluiu-se que os designers visuais podem explorar esse campo, a fim de contribuir no projeto destes materiais educacionais.

Palavras-chave: Revisão de Literatura, Design Emocional, Design Instrucional, Aprendizagem Multimídia, Projeto de Materiais Multimídia.
Abstract: Emotions are present in all daily activities, including learning. This paper presents a systematic literature review about the relationship between emotions and learning for the design of multimedia educational materials. From the databases Web of Science and Periódicos CAPES, articles internationally published from 2010 to 2015 were searched. After data analysis, it was possible to notice that few studies have been developed in the past few years, but the ones published are valuable, for in overview these studies confirm the emotions influence on learning and the importance of evoking emotions that motivate students facilitating the use of their cognitive capacity as good as possible. In these articles, it is 
indicated the importance and influence of graphic-visual elements of the materials, therefore, since these elements evoke emotions in the students, it was concluded that visual designers can explore this field in order to contribute to the project of these educational materials.

Keywords: Literature Review, Emotional Design, Instructional Design, Multimedia Learning, Multimedia Design.

\section{INTRODUÇÃO}

A área do design emocional vem estudando aspectos dos produtos utilizados diariamente. São abordados os motivos pelos quais gostamos ou não de determinados produtos. Anteriormente à emergência desse campo, o foco dos estudos encontravase sobre a usabilidade, que tradicionalmente destaca a facilidade de uso e a funcionalidade (NORMAN, 2008). No presente, a comunidade do design emocional concentra-se em entender como projetar visando evocar ou evitar determinadas emoções, a partir do entendimento das necessidades do usuário (TONETTO; COSTA, 2011). Contudo, quando se trata de produtos voltados à aprendizagem, este interesse não ocorre da mesma forma. Estudos científicos relacionando o design emocional e a educação são recentes (MAYER; ESTRELLA, 2014).

Os materiais instrucionais foram baseados apenas em textos. Sabe-se que a apresentação de imagens complementares aos textos favorece a aprendizagem, o que fundamenta o princípio multimídia (MAYER, 2005). Desta forma, a aprendizagem baseada em tecnologia, através de mídias digitais, vem se tornando cada vez mais disponível e desejável.

As emoções podem ser entendidas como reações humanas a estímulos relevantes. Em relação à aprendizagem, podem estar envolvidas em situações individuais ou sociais, como por exemplo, a realização de uma tarefa individual em casa ou a instrução em sala de aula (PEKRUN, 1992).

Fialho (2001) afirma que as emoções ativam objetivos definidos como pensar e agir. Ou seja, a partir de um estímulo emocional, o ser humano raciocina e toma uma decisão. Norman (2008) corrobora com essa afirmação ao explicar que as emoções, tanto positivas como negativas, são importantes na tomada de decisão. 0 autor enfatiza a influência das emoções positivas em relação ao aprendizado, à curiosidade e ao pensamento positivo.

Sendo assim, constata-se a necessidade de compreender como o design emocional pode estar relacionado às questões pertinentes à aprendizagem baseada em materiais educacionais multimídia, e, consequentemente, como deve ser abordado no projeto desses materiais. Este trabalho traz breves considerações teóricas que fundamentam a necessidade dos estudos que tratam da relação entre aprendizagem e emoções e uma revisão dos artigos publicados internacionalmente no período de 2010 a 2015 que abordam temas pertinentes às relações das emoções e o projeto de materiais educacionais multimídia. 


\section{EMOÇÕES, COGNIÇÃO E APRENDIZAGEM MULTIMÍDIA}

Quando falamos em emoções, estamos tratando também de afetos e humores. Tais termos frequentemente são entendidos como sinônimos, porém, possuem relevantes diferenças. Emoções são caracterizadas por apresentarem diferentes perfis, algumas são ativadas em um padrão de "explosão", com início rápido, auge de intensidade e declínio acelerado, outras possuem padrão "ondulatório", podendo variar de perfil de acordo com as características e o contexto do usuário. Quando estados emocionais se prolongam por extensos períodos de tempo, podemos nomeálos como humores. O termo afeto caracteriza o que é expresso ou manifestado em relação a um objeto ou acontecimento, em qualquer momento, independente do estado de humor (DAMÁSIO, 2000).

Nos itens a seguir são apresentadas considerações teóricas acerca dos estudos que embasam a relação das emoções, cognição e aprendizagem multimídia.

\subsection{A Relação entre Emoções, Afeto e Aprendiz}

Nos seus estudos iniciais, Damásio (2000) estabelece que as emoções estão relacionadas à conservação da vida pelo organismo e são definidas como processos determinados biologicamente, dependentes de mecanismos cerebrais que regulam e representam estados corporais. Esses processos regulatórios são automáticos e estão relacionados a tudo que envolve nosso organismo. Isso posto, considera-se que os estados afetivos gerados influenciam também os processos cognitivos, atuando diretamente na forma como aprendemos.

Emoções positivas e negativas podem ser desencadeadas e envolvidas em uma situação de aprendizagem. Estudos apontam que, durante a aprendizagem, as informações que foram assimiladas em um determinado estado emocional são armazenadas junto às memórias do mesmo estado emocional. Ainda, essas informações são melhor recuperadas quando o estudante apresenta o mesmo humor manifestado no momento no qual aprendeu o conteúdo (PEKRUN, 1992).

Afeto e cognição atuam em interdependência, ou seja, assim como os processos cognitivos determinam reações emocionais, os estados afetivos influenciam a forma como as pessoas percebem, interpretam e recordam situações. Dessa forma, emoções, afetos e humores são intrínsecos ao processamento e recuperação das informações apresentadas nos materiais educacionais de qualquer tipo (FORGAS, 2008).

\subsection{As Emoções e as Teorias Cognitivas}

Mayer (2005), ao apresentar a teoria cognitiva de aprendizagem multimídia, considera que a aprendizagem significativa depende do engajamento no processamento cognitivo adequado durante a aprendizagem. Trata-se de um estudo sobre como as pessoas aprendem a partir de palavras e imagens, com base na ideia de que possuímos canais separados para processamento de informações verbais e visuais, e que cada um desses canais possui capacidade limitada para processamento de materiais a cada vez.

A tomada de decisões quanto a tarefa que está sendo realizada, quando decide-se continuar ou abandonar o que se está fazendo ou dedicar-se mais ou menos, 
está compreendida pela regulação das atividades, que é mediada pela relação entre afeto e cognição (FIALHO, 2001). Isso posto, compreende-se que as emoções não podem ser menosprezadas nas teorias que abordam o processamento cognitivo.

Em sequência da teoria cognitiva de aprendizagem multimídia de Mayer (2005), Moreno e Mayer (2007) propuseram a teoria cognitiva-afetiva de aprendizagem multimídia, onde indicam o papel da motivação e do afeto no processo cognitivo. Considera-se a ideia de que fatores motivacionais e afetivos medeiam a aprendizagem, influenciando o grau de envolvimento cognitivo.

Este modelo (Figura 1) prevê que o aluno esteja em contato com um material multimídia contendo textos escritos ou falados, combinados com imagens ou sons, elementos os quais são processados pela memória sensorial através dos ouvidos e olhos. A partir dessa entrada, o aluno atentaria e selecionaria as informações relevantes e as processaria através da memória de trabalho, que consiste em um estado consciente de processamento do conhecimento, fase mais importante para a aprendizagem multimídia. Em seguida, organizaria as representações obtidas em um modelo mental coerente e o relacionaria com o conhecimento prévio. É previsto que o aluno utilizasse suas habilidades metacognitivas durante esse processamento, a fim de controlar a motivação e o processamento cognitivo (MAYER, 2005; MORENO, 2006; MORENO; MAYER, 2007).

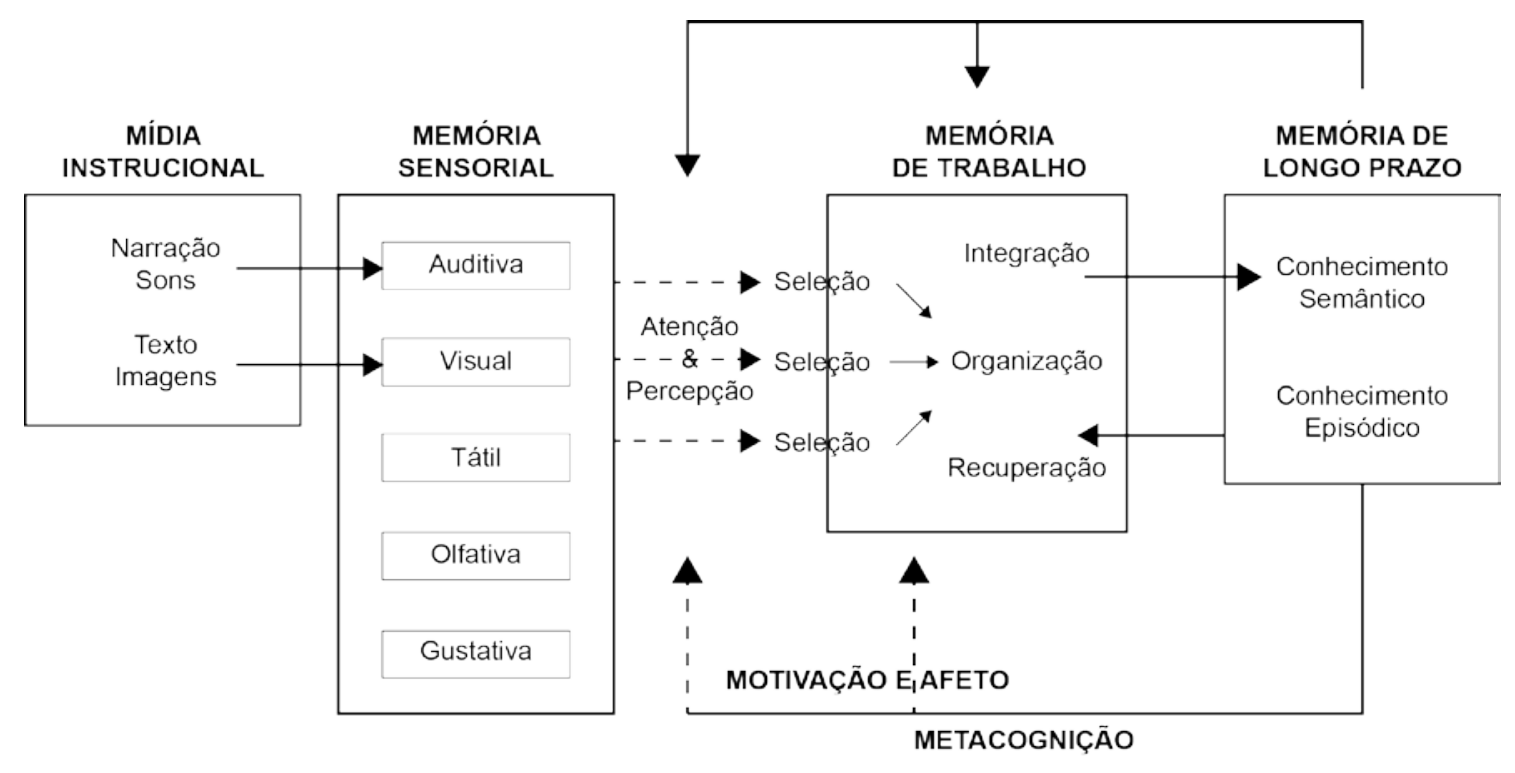

Figura 1 - Modelo cognitivo-afetivo de aprendizagem através de mídia.

Fonte: Adaptado de Moreno e Mayer (2007).

\section{MÉTODO DE PESQUISA}

O presente estudo foi executado na forma de uma revisão bibliográfica sistemática, baseada nos procedimentos do método RBS Roadmap. Trata-se de um roteiro sistematizado para a busca, identificação, classificação e análise de artigos científicos que proporciona a visão do estado da arte do tema em foco (CONFORTO; AMARAL; SILVA, 2011). 


\subsection{Definição do Problema}

A realização desse estudo se origina na necessidade de identificar o corpo de conhecimento do campo do design emocional em relação à aprendizagem baseada em materiais educacionais multimídia. O estudo tem como objetivo conhecer as teorias que lastreiam as decisões de projeto desses materiais, considerando o viés emocional.

\subsection{Busca dos Artigos}

A busca dos artigos foi efetuada em bases de dados internacionais (Web of Science e Periódicos CAPES) escolhidas por contemplarem um grande número de periódicos. Para tanto, foram utilizadas as strings "multimedia learning" AND "emotional design" e "multimedia learning" AND "emotion". Foi indicado o período dos 5 últimos anos, de 2010 a 2015, com a finalidade de conhecer as publicações mais recentes e entender como o tema está sendo abordado nesse recorte.

\subsection{Seleção e Avaliação Crítica dos Artigos}

A partir de leitura preliminar do título e resumo, os artigos foram selecionados com a utilização de critérios elaborados para a inclusão dos mesmos. Os artigos deveriam tratar necessariamente sobre estudos práticos (com base em experimentos) e atender a, pelo menos, um dos seguintes critérios: (i) estudos que abordam as emoções relacionadas à aprendizagem baseada em materiais de aprendizagem multimídia; (ii) artigos que tratam de tópicos específicos de projeto dos materiais multimídia em relação às emoções; (iii) Artigos nos idiomas português ou inglês.

O somatório dos resultados gerais das buscas totalizou 119, porém, apenas 10 artigos foram selecionados. Os resultados excluídos não atendiam aos critérios de inclusão, eram repetidos ou abordavam tópicos de outras áreas do conhecimento. Os artigos selecionados foram catalogados em software específico (Mendeley) e listados em planilha eletrônica.

\subsection{Coleta e Análise dos Dados}

Foram lidos os 10 artigos que compõe a amostra do presente estudo e, a partir do teor dos mesmos, analisados e classificados quanto (i) à área de conhecimento dos periódicos, (ii) ao tema abordado, (iii) às ferramentas utilizadas para identificação das emoções, (iv) aos tipos de materiais multimídia utilizados nos experimentos, (v) aos tipos de emoções discutidas, (vi) ao nível escolar dos alunos envolvidos, (vii) ao país onde foram realizados os experimentos.

\section{RESULTADOS}

Nos itens seguintes, em quadros, são apresentados os resultados obtidos a partir da análise dos dados coletados.

\section{1 Área de Conhecimento dos Periódicos Encontrados}

Os 10 trabalhos selecionados estão publicados em 6 diferentes periódicos, das áreas de Educação/Instrução, Computação e Psicologia (Quadro 1).

É importante observar que não foram encontradas publicações em periódicos específicos do campo do Design. 
Quadro 1 - Artigos organizados de acordo com o periódico e ano da publicação.

\begin{tabular}{|c|c|c|c|c|}
\hline Periódico & Título & Autores & Ano & Palavras-chave \\
\hline \multirow{2}{*}{$\begin{array}{l}\text { Computers } \\
\text { \& Education }\end{array}$} & $\begin{array}{l}\text { Assessing the effects of different } \\
\text { multimedia materials on emotions } \\
\text { and learning performance for visual } \\
\text { and verbal style learners }\end{array}$ & $\begin{array}{l}\text { Chen, C.; } \\
\text { Sun, Y. }\end{array}$ & 2012 & $\begin{array}{l}\text { Media in education, } \\
\text { Multimedia/hypermed } \\
\text { ia systems, Evaluation } \\
\text { methodologies. } \\
\end{array}$ \\
\hline & $\begin{array}{l}\text { Emotional design and positive } \\
\text { emotions in multimedia learning: } \\
\text { An eyetracking study on the use of } \\
\text { anthropomorphisms }\end{array}$ & $\begin{array}{l}\text { Park, B.; } \\
\text { Knorzer, L.; } \\
\text { Plass, J. L.; } \\
\text { Brünken, R. }\end{array}$ & 2015 & $\begin{array}{l}\text { Multimedia learning, } \\
\text { Eyetracking, CATLM, } \\
\text { Emotion induction, } \\
\text { Positive emotions. }\end{array}$ \\
\hline \multirow{3}{*}{$\begin{array}{l}\text { Computers } \\
\text { in Human } \\
\text { Behavior }\end{array}$} & $\begin{array}{l}\text { Cognitive and affective effects of } \\
\text { seductive details in multimedia } \\
\text { learning }\end{array}$ & $\begin{array}{l}\text { Park, B.; } \\
\text { Flowerday, } \\
\text { T.; } \\
\text { Brünken, R. }\end{array}$ & 2014 & $\begin{array}{l}\text { Multimedia learning, } \\
\text { Seductive details. }\end{array}$ \\
\hline & $\begin{array}{l}\text { The effects of various multimedia } \\
\text { instructional materials on students' } \\
\text { learning responses and outcomes: } \\
\text { A comparative experimental study }\end{array}$ & $\begin{array}{l}\text { Lee, Y.; } \\
\text { Hsiao, C.; } \\
\text { Ho, C. }\end{array}$ & 2014 & $\begin{array}{l}\text { Animated characters, } \\
\text { Multimedia } \\
\text { instructional materials, } \\
\text { Flow experience, } \\
\text { Socialness } \\
\text { perceptions, Learning } \\
\text { motivation, Learning } \\
\text { outcome. }\end{array}$ \\
\hline & $\begin{array}{l}\text { Emotional design in multimedia } \\
\text { learning: Differentiation on } \\
\text { relevant design features and their } \\
\text { effects on emotions and learning }\end{array}$ & $\begin{array}{l}\text { Heidig, S.; } \\
\text { Müller, J.; } \\
\text { Reichelt, M. }\end{array}$ & 2015 & $\begin{array}{l}\text { Multimedia learning, } \\
\text { Emotional design, } \\
\text { Emotion Aesthetics, } \\
\text { Usability. }\end{array}$ \\
\hline $\begin{array}{l}\text { Instructional } \\
\text { Science }\end{array}$ & $\begin{array}{l}\text { Emotion and multimedia learning: } \\
\text { an investigation of the effects of } \\
\text { valence and arousal on different } \\
\text { modalities in an instructional } \\
\text { animation }\end{array}$ & $\begin{array}{l}\text { Chung, S.; } \\
\text { Cheon, J.; } \\
\text { Lee, K. }\end{array}$ & 2015 & $\begin{array}{l}\text { Multimedia learning, } \\
\text { Emotion, Motivated } \\
\text { cognition, Limited } \\
\text { capacity model of } \\
\text { motivated mediated } \\
\text { message processing, } \\
\text { LC4MP. }\end{array}$ \\
\hline $\begin{array}{l}\text { Journal of } \\
\text { Educational } \\
\text { Psychology }\end{array}$ & $\begin{array}{l}\text { Emotional Design in Multimedia } \\
\text { Learning }\end{array}$ & $\begin{array}{l}\text { Um, E. R.; } \\
\text { Plass, J. L.; } \\
\text { Hayward, } \\
\text { E.O.; } \\
\text { Homer, B. D. }\end{array}$ & 2011 & $\begin{array}{l}\text { Emotion, Multimedia } \\
\text { learning, Cognitive } \\
\text { load, Motivation, } \\
\text { Instructional design }\end{array}$ \\
\hline \multirow[b]{2}{*}{$\begin{array}{l}\text { Learning and } \\
\text { Instruction }\end{array}$} & $\begin{array}{l}\text { Benefits of emotional design in } \\
\text { multimedia instruction }\end{array}$ & $\begin{array}{l}\text { Mayer, R.; } \\
\text { Estrella, G. }\end{array}$ & 2014 & $\begin{array}{l}\text { Multimedia learning, } \\
\text { Emotional design, } \\
\text { Motivation. }\end{array}$ \\
\hline & $\begin{array}{l}\text { Emotional design in multimedia } \\
\text { learning: Effects of shape and color } \\
\text { on affect and learning }\end{array}$ & $\begin{array}{l}\text { Plass, J. L.; } \\
\text { Heidig, S.; } \\
\text { Hayward, E. } \\
\text { O.; } \\
\text { Homer, B. D.; } \\
\text { Um, E. }\end{array}$ & 2014 & $\begin{array}{l}\text { Emotion, Emotional } \\
\text { design, Learning } \\
\text { Multimedia, Cognition. }\end{array}$ \\
\hline $\begin{array}{l}\text { Library \& } \\
\text { Information } \\
\text { Science } \\
\text { Research }\end{array}$ & $\begin{array}{l}\text { Using emotion recognition } \\
\text { technology to assess the effects of } \\
\text { different multimedia materials on } \\
\text { learning emotion and performance }\end{array}$ & $\begin{array}{l}\text { Chen, C.,; } \\
\text { Wang, H. }\end{array}$ & 2011 & Não apresenta. \\
\hline
\end{tabular}

Fonte: Elaborado pelos autores, com base na pesquisa realizada. 


\subsection{Classificação dos Estudos}

Conforme apresentado no Quadro 2, os estudos foram realizados em diferentes contextos, utilizando diferentes materiais multimídia. Dos 10 estudos analisados, 8 experimentos envolveram alunos de ensino superior e 2 experimentos envolveram alunos de ensino básico. Também foram utilizados diferentes tipos de ferramentas de identificação ou medição das emoções. Nem todos os artigos informam precisamente em qual país os experimentos foram executados, os que foram identificados concentram-se na Alemanha (3), em Taiwan (3), e nos Estados Unidos (2).

Quadro 2 - Classificação dos estudos.

\begin{tabular}{|c|c|c|c|c|c|c|}
\hline AUTORES & TEMA & $\begin{array}{l}\text { FERRAMENTAS DE } \\
\text { IDENTIFICAÇÃO OU } \\
\text { MEDIÇÃO DAS } \\
\text { EMOÇÕES }\end{array}$ & $\begin{array}{l}\text { MATERIAIS } \\
\text { UTILIZADOS } \\
\text { NOS } \\
\text { EXPERIMENTOS }\end{array}$ & EMOÇÕES & $\begin{array}{l}\text { NÍVEL } \\
\text { ESCOLAR }\end{array}$ & PAÍS \\
\hline $\begin{array}{l}\text { Chen, C.; } \\
\text { Wang, H., } \\
2011\end{array}$ & $\begin{array}{l}\text { Diferentes } \\
\text { tipos de } \\
\text { materiais. }\end{array}$ & $\begin{array}{l}\text { emWave System } \\
\text { (Detector de } \\
\text { tensões para } \\
\text { estados } \\
\text { emocionais. } \\
\text { HeartMath } \\
\text { Institute). }\end{array}$ & $\begin{array}{l}\text { Texto e } \\
\text { imagem } \\
\text { estáticos; } \\
\text { Vídeo; } \\
\text { Animação } \\
\text { interativa. }\end{array}$ & $\begin{array}{l}\text { Positivas e } \\
\text { Negativas }\end{array}$ & Básico & Taiwan \\
\hline $\begin{array}{l}\text { Um, E. R.; } \\
\text { Plass, J. L.; } \\
\text { Hayward, } \\
\text { E. O.; } \\
\text { Homer, B. } \\
\text { D., } 2011\end{array}$ & $\begin{array}{l}\text { Elementos } \\
\text { visuais: } \\
\text { cores e } \\
\text { formas. }\end{array}$ & $\begin{array}{l}\text { PAS - Positive } \\
\text { Affect Scale } \\
\text { (PANAS. Watson; } \\
\text { Clark; Tellegen, } \\
\text { 1988). }\end{array}$ & $\begin{array}{l}\text { Lição } \\
\text { multimídia } \\
\text { navegável, } \\
\text { contendo } \\
\text { animações. }\end{array}$ & Positivas & Superior & $\begin{array}{l}\text { Estados } \\
\text { Unidos }\end{array}$ \\
\hline $\begin{array}{l}\text { Chen, C.; } \\
\text { Sun, Y., } \\
2012\end{array}$ & $\begin{array}{l}\text { Estilos } \\
\text { cognitivos } \\
\text { dos } \\
\text { estudantes: } \\
\text { verbalizador } \\
\text { es e } \\
\text { visualizador } \\
\text { es. }\end{array}$ & $\begin{array}{l}\text { emWave System } \\
\text { (Detector de } \\
\text { tensões para } \\
\text { estados } \\
\text { emocionais. } \\
\text { HeartMath } \\
\text { Institute). }\end{array}$ & $\begin{array}{l}\text { Texto e } \\
\text { imagem } \\
\text { estáticos; } \\
\text { Vídeo; } \\
\text { Animação } \\
\text { interativa. }\end{array}$ & $\begin{array}{l}\text { Positivas e } \\
\text { Negativas }\end{array}$ & Básico & Taiwan \\
\hline $\begin{array}{l}\text { Plass, J. L.; } \\
\text { Heidig, S.; } \\
\text { Hayward, } \\
\text { E. O.; } \\
\text { Homer, B. } \\
\text { D.; Um, E., } \\
2014\end{array}$ & $\begin{array}{l}\text { Elementos } \\
\text { visuais: } \\
\text { cores e } \\
\text { formas. }\end{array}$ & $\begin{array}{l}\text { PAS - Positive } \\
\text { Affect Scale } \\
\text { (PANAS. Watson; } \\
\text { Clark; Tellegen, } \\
\text { 1988). }\end{array}$ & $\begin{array}{l}\text { Lição } \\
\text { multimídia } \\
\text { navegável, } \\
\text { contendo } \\
\text { animações. }\end{array}$ & Positivas & Superior & $\begin{array}{l}\text { Alema- } \\
\text { nha }\end{array}$ \\
\hline $\begin{array}{l}\text { Lee, Y.; } \\
\text { Hsiao, C.; } \\
\text { Ho, C., } \\
2014\end{array}$ & $\begin{array}{l}\text { Estilos de } \\
\text { apresentaçã } \\
\text { o. }\end{array}$ & $\begin{array}{l}\text { Classificação em } \\
\text { escala de } 5 \\
\text { pontos a partir de } \\
\text { questões. }\end{array}$ & $\begin{array}{l}\text { Apresentação } \\
\text { em } \\
\text { Powerpoint } \\
\text { simples ou } \\
\text { guiada por } \\
\text { diferentes } \\
\text { pesonagens } \\
\text { (humano ou } \\
\text { monstro). }\end{array}$ & $\begin{array}{l}\text { Positivas e } \\
\text { Negativas }\end{array}$ & Superior & Taiwan \\
\hline $\begin{array}{l}\text { Mayer, R.; } \\
\text { Estrella, } \\
\text { G., } 2014\end{array}$ & $\begin{array}{l}\text { Cores e } \\
\text { formas. }\end{array}$ & - & $\begin{array}{l}\text { Apresentação } \\
\text { em } \\
\text { Powerpoint. }\end{array}$ & $\begin{array}{l}\text { Não } \\
\text { indicadas }\end{array}$ & Superior & $\begin{array}{l}\text { Estados } \\
\text { Unidos }\end{array}$ \\
\hline Park, B.; & Detalhes & PANAS-X General & Telas com & Positivas & Superior & - \\
\hline
\end{tabular}




\begin{tabular}{|c|c|c|c|c|c|c|}
\hline $\begin{array}{l}\text { Flowerday, } \\
\text { T; } \\
\text { Brünken, } \\
\text { R., } 2014\end{array}$ & $\begin{array}{l}\text { Sedutores: } \\
\text { informações } \\
\text { adicionais ao } \\
\text { conteúdo } \\
\text { básico. }\end{array}$ & $\begin{array}{l}\text { Dimension Scale } \\
\text { (Watson; Clark, } \\
\text { 1994). }\end{array}$ & $\begin{array}{l}\text { conteúdo } \\
\text { multimídia } \\
\text { (imagens e } \\
\text { equivalência } \\
\text { verbal). }\end{array}$ & & & \\
\hline $\begin{array}{l}\text { Heidig, S.; } \\
\text { Müller, J; } \\
\text { Reichelt, } \\
\text { M., } 2015\end{array}$ & $\begin{array}{l}\text { Característic } \\
\text { as estéticas } \\
\text { e } \\
\text { usabilidade. }\end{array}$ & $\begin{array}{l}\text { Scales for } \\
\text { Assessing } \\
\text { Positive/ } \\
\text { Negative } \\
\text { Activation and } \\
\text { Valence in } \\
\text { Experience } \\
\text { Sampling Studies } \\
\text { (PANAVA-KS; } \\
\text { Schallberger, } \\
\text { 2005). }\end{array}$ & $\begin{array}{l}\text { Material } \\
\text { multimídia } \\
\text { interativo. }\end{array}$ & $\begin{array}{l}\text { Positivas e } \\
\text { Negativas }\end{array}$ & Superior & $\begin{array}{l}\text { Alema- } \\
\text { nha }\end{array}$ \\
\hline $\begin{array}{l}\text { Chung, S.; } \\
\text { Cheon, J.; } \\
\text { Lee, K., } \\
2015\end{array}$ & $\begin{array}{l}\text { Influência da } \\
\text { valência e } \\
\text { níveis de } \\
\text { excitação. }\end{array}$ & $\begin{array}{l}\text { Classificação em } \\
\text { escala de } 9 \\
\text { pontos a partir de } \\
\text { questões. }\end{array}$ & $\begin{array}{l}\text { Vídeos (para } \\
\text { indução de } \\
\text { emoções) e } \\
\text { animação } \\
\text { instrucional. }\end{array}$ & $\begin{array}{l}\text { Positivas e } \\
\text { Negativas }\end{array}$ & Superior & - \\
\hline $\begin{array}{l}\text { Park, B.; } \\
\text { Knorzer, L.; } \\
\text { Plass, J. L.; } \\
\text { Brünken, } \\
\text { R., } 2015\end{array}$ & $\begin{array}{l}\text { Antropomor } \\
\text { fis-mo. }\end{array}$ & $\begin{array}{l}\text { PAS - Positive } \\
\text { Affect Scale } \\
\text { (PANAS. Watson; } \\
\text { Clark; Tellegen, } \\
\text { 1988). }\end{array}$ & $\begin{array}{l}\text { Lição } \\
\text { multimídia } \\
\text { navegável, } \\
\text { contendo } \\
\text { animações. }\end{array}$ & Positivas & Superior & $\begin{array}{l}\text { Alema- } \\
\text { nha }\end{array}$ \\
\hline
\end{tabular}

Fonte: Elaborado pelos autores, com base na pesquisa realizada.

\section{DISCUSSÃO DOS RESULTADOS}

Os estudos trazem experimentos realizados a partir do contexto apresentado no Quadro 2. Os experimentos validam ou refutam hipóteses sobre questões que relacionam as emoções e aprendizagem através dos materiais de aprendizagem multimídia e podem dar suporte às escolhas ao projetar tais materiais.

É importante observar que os estudos analisados sugerem que os resultados podem não ser passíveis de generalização e devem sofrer alterações de acordo com o perfil dos alunos em termos culturais, de gênero e conhecimento prévio, por exemplo.

\subsection{As Emoções no Projeto de Materiais Educacionais Multimídia}

Heidig, Muller e Reichelt (2015), apontam que na área de multimídia para a aprendizagem, assim como na área de interação humano-computador em geral e particularmente Webdesign, o foco dos estudos tem sido a usabilidade, em detrimento de questões que envolvem a beleza, estética, prazer e diversão. Porém, estes tópicos vêm se apresentando quando se trata da experiência do usuário como um todo.

O design emocional de materiais educacionais multimídia contempla o uso de elementos de design visual que podem evocar emoções positivas. Não se trata necessariamente de elementos adicionais, mas de elementos intrínsecos, como cor, forma ou layout (HEIDIG; MULLER; REICHELT, 2015). Aumentar a atratividade de gráficos presentes no conteúdo a ser instruído tem efeito positivo na aprendizagem.

Estudos (Um et al., 2011; Plass et al., 2014) indicam que os designers podem usar cores quentes ao invés de cores frias e formas que lembrem rostos humanos 
(redondas em vez de quadradas) em seus projetos, a fim de induzir emoções positivas nos alunos. Estudos similares (Mayer; Estrella, 2014; Park et al., 2015), observam que esses princípios devem ser utilizados nos gráficos relevantes para o objetivo instrucional, a fim de concentrar a atenção dos alunos no conteúdo essencial, com cuidado para não os distrair com conteúdos irrelevantes. Os elementos que são interessantes, mas não fazem parte do conteúdo básico de uma lição, denominados "detalhes sedutores", são tratados na pesquisa de Park, Flowerday e Brunken (2014).

O tipo de multimídia apresentado aos alunos também constitui fator importante no design emocional voltado à aprendizagem. Chen e Wang (2011) constataram interessantes resultados com seus experimentos. Utilizando um sensor que detecta tensões emocionais, os alunos foram expostos a diferentes materiais multimídias com o mesmo conteúdo (um texto estático combinado com imagens, um vídeo e uma animação interativa). Os autores concluíram, preliminarmente, que o material no formato de vídeo gerou reações emocionais positivas mais intensas em relação aos demais, favorecendo melhor desempenho na aprendizagem. Os demais materiais suscitaram emoções negativas por mais vezes durante a utilização, indicando que o interesse dos alunos foi menor comparada a utilização do vídeo. Também foi percebido que as estudantes do sexo feminino são mais afetadas pelas diferenças dos materiais do que os estudantes do sexo masculino.

A partir desse panorama geral dos trabalhos componentes da amostra, é possível perceber que os estudos foram relevantes e é interessante que sejam repetidos com outros grupos, em outros contextos.

\section{CONCLUSÃO}

A revisão de literatura revela que a relação do design emocional com a aprendizagem baseada em materiais educacionais multimídia é recente e que apresenta poucas publicações na área. Os resultados demonstraram que os pesquisadores, em grande parte, são autores de mais de um trabalho e que, ao longo dos últimos cinco anos, deram continuidade aos experimentos devido a necessidade de mais estudos acerca de diferentes temas e enfoques dentro da área.

De forma geral, os resultados encontrados são consistentes com a teoria cognitiva-afetiva de aprendizagem multimídia de Mayer e Moreno (2007), corroborando com a ideia de que os estudantes regulam o processo cognitivo através das reações emocionais e reforçando o importante papel da motivação. Fica explícita a influência das emoções na aprendizagem e a necessidade de pesquisas sobre como suscitar emoções que motivem os alunos e gerem o melhor uso de sua capacidade cognitiva.

Nota-se também que profissionais do campo do Design Visual e Design Instrucional, podem explorar e contribuir com estudos sobre a relação das emoções e os materiais educacionais multimídia, visto que o tratamento visual dos materiais pode induzir emoções nos alunos, influenciando o interesse pelo conteúdo e o processamento cognitivo. Considerando-se que as publicações encontradas se concentram em periódicos de outras áreas do conhecimento (Educação/Instrução, Computação e Psicologia), é interessante difundir a temática dentre a comunidade do Design. 


\section{REFERÊNCIAS}

CHEN, C.; WANG, H. Using emotion recognition technology to assess the effects of different multimedia materials on learning emotion and performance. Library \& Information Science Research, v. 33(3), p. 244-255, 2011.

CHEN, C.; SUN, Y. Assessing the effects of different multimedia materials on emotions and learning performance for visual and verbal style learners. Computers and Education, v. 59(4), p. 1273-1285, 2012.

CHUNG, S.; CHEON, J.; LEE, K. Emotion and multimedia learning: an investigation of the effects of valence and arousal on different modalities in an instructional animation. Instructional Science, 43(5), 545-559, 2015.

CONFORTO, E. C.; AMARAL, D. C.; SILVA, S. L. DA. Roteiro para revisão bibliográfica sistemática: aplicação no desenvolvimento de produtos e gerenciamento de projetos. In: $8^{\circ}$ Congresso Brasileiro de Gestão de Desenvolvimento de Produto - CBGDP 2011. Porto Alegre, RS, Brasil. Instituto de Gestão de Desenvolvimento do Produto - IGDP. Anais... Porto Alegre: IGDP, 2011.

DAMÁSIO, A. O mistério da consciência: do corpo e das emoções ao conhecimento de si. São Paulo: Companhia das Letras, 2000.

FIALHO, F. A. P. Introdução às ciências da cognicão. 10 Ed. Florianópolis: Insular, 2001. FORGAS, J. P. Affect and cognition. Perspectives on Psychological Science, v. 3, p. 94101, 2008.

HEARTHMATH INSTITUTE. EmWave Pro. Disponível na internet por http em: $<$ http://store.heartmath.org/emWave-PC/emwave-pro.html>. Acesso em jan. 2016.

HEIDIG, S.; MÜLLER, J.; REICHELT, M. Emotional design in multimedia learning: Differentiation on relevant design features and their effects on emotions and learning. Computers in Human Behavior, v. 44, p. 81-95, 2015.

LEE, Y.; HSIAO, C.; HO, C. The effects of various multimedia instructional materials on students' learning responses and outcomes: A comparative experimental study. Computers in Human Behavior, v. 40, p. 119-132, 2014.

MAYER, R. E. Cognitive theory of multimedia learning. In: R. E. Mayer (Ed.), The Cambridge handbook of multimedia learning (p. 31-48). Cambridge: Cambridge University Press, 2005.

MAYER, R. E.; ESTRELLA, G. Benefits of emotional design in multimedia instruction. Learning and Instruction, v. 33, p. 12-18, 2014.

MORENO, R. Does the modality principle hold for different media? A test of the method-affects-learning hypothesis. Journal of Computer Assisted Learning, v. 22, p. 149-158, 2006.

MORENO, R.; MAYER, R. E. Interactive multimodal learning environments. Educational Psychology Review, v. 19, p. 309-326, 2007.

NORMAN, D. A. Design Emocional: por que adoramos (ou detestamos) os objetos do dia-a-dia. Rio de Janeiro: Rocco, 2008. 
PARK, B.; FLOWERDAY, T.; BRÜNKEN, R. Cognitive and affective effects of seductive details in multimedia learning. Computers in Human Behavior, v. 44, p. 267-278, 2015.

PARK, B. et. al. Emotional design and positive emotions in multimedia learning: An eyetracking study on the use of anthropomorphisms. Computers and Education, v. 86, p. 30-42, 2015.

PEKRUN, R. The impact of emotions on learning and achievement: Towards a theory of cognitive/motivational mediators. Applied Psychology, v. 41, p. 359-376, 1992.

PLASS, J. L. et. al. Emotional design in multimedia learning: effects of shape and color on affect and learning. Learning and Instruction, v. 29, p. 128-140, 2014.

SCHALLBERGER, U. Kurzskalen zur Erfassung der Positiven Aktivierung, Negativen Aktivierung und Valenz in Experience Sampling Studien (PANAVA- KS). Theoretische und methodische Grundlagen, Konstruktvalidität und psychometrische Eigenschaften bei der Beschreibung intraund interindividueller Unterschiede. Forschungsberichte aus dem Projekt: (Qualität des Erlebens in Arbeit und Freizeit), Nr. 6. Zürich: Fachrichtung Angewandte Psychologie des Psychologischen Instituts der Universität, 2005.

TONETTO, L.; COSTA, F. Design emocional: conceitos, abordagens e perspectivas de pesquisa. Strategic Design Research Journal, v. 4 (3), p. 132-140, 2011.

UM, E. et. al. Emotional design in multimedia learning. Journal of Educational Psychology, v. 104 (2), p. 485-498, 2011.

WATSON, D.; CLARK, L. A.; TELLEGEN, A. Development and validation of brief measures of positive and negative affect: the PANAS scales. Journal of Personality and Social Psychology, v. 54 (6), p. 1063-1070, 1998.

WATSON, D.; CLARK, L. A. Manual for the positive and negative affect schedule Expanded form. University of lowa, 1994. 